1 Theon, J. S., Nordberg, W., and Smith, W. S., Science, N.Y. 157, 419 (1967).

2 Hesstvedt, E., Space Res., 9, 170 (1969).

3 Witt, G., Space Res., 9, 157 (1969).

${ }^{4}$ Hamilton, R. A., Met. Mag. Lond., 93, 201 (1964).

5 Scott, A. F. D., COSPAR Meeting, Konstanz (June 1973).

${ }^{6}$ Smith, W. S., Theon, J. S., Casey, J. F., Azcarraga, A., and

Horvath, J. J., NASA TR-R-360 (Washington, DC 1971).

7 Paton, J., Met. Mag. Lond., 93-102 (1964-1973).

8 Staff, Stratospheric Research Group, Free University Berlin, Meteor. Abh. Berlin, (1959-73).

${ }_{9}^{9}$ Labitske, K., and collaborators, Climatology of the Stratosphere in the Northern Hemisphere, Meteor. Abh. Berlin, 100, No. 4 (1972).

\section{Electric cloud and weather modification with intense relativistic electron beams}

THE problems confronting weather modification by seeding methods are well known and centre on the dispersion of the seeding agent over a large air volume. In a different method for artificial cloud modification proposed by Vonnegut and Moore $^{1}$ electric charges are released by corona discharges into updraughts from the ground by a system of wires at a high electric potential. In this method the electric charges are much more rapidly dispersed, due to a rather large ion mobility in a thundercloud electric field, than is otherwise possible for seeding agents dispersed by turbulent convection. But the system of ground wires seems to be impractical for a weather modification technique with the demand for high mobility.

I therefore propose here an alternative method of electric cloud and weather modification based on the projection of a rapid sequence of intense relativistic electron beams into the atmosphere. The maximum range in the atmosphere of relativistic electrons is given by the radiation length $l_{0} \sim$ $280 \mathrm{~m}$ and which is rapidly approached for electron energies in excess of $50 \mathrm{MeV}$. A beam of electrons would normally disperse rapidly in the radial direction due to multiple scattering. This however, is not the case for an intense beam of relativistic electrons which is confined by its own magnetic field. Beams up to $10^{6} \mathrm{~A}$ and with electron energies of many million electron volts lasting up to $100 \mathrm{~ns}$ have been already produced by the combination of a Marx surge generator with a Blumlein transmission line, connected to a field emission diode. The total beam energies so far achieved reach the value of megajoules. If such a beam is projected into the atmosphere it will not disperse radially but rather will produce a channel of hot air, with a maximum channel length equal to the radiation length in air. If now a second beam is projected into this channel after a time lapse which is equal to the time for the air to expand in the channel heated by the first beam and which is given by $\tau_{\exp } \sim r / a$, where $r$ is the beam radius and $a$ the velocity of sound in the heated air, then the second beam will in part propagate through air of reduced density due to the thermal expansion caused by the first beam. If for example, the first beam heats the channel up to $\sim 10^{4} \mathrm{~K}$ the velocity of sound would be $\sim$ $10^{5} \mathrm{~cm} \mathrm{~s}^{-1}$, and if the original beam radius is $r \sim 0.1 \mathrm{~cm}$ the time for expansion would be $\tau_{\exp } \sim 10^{-6} \mathrm{~s}$. Because of the reduced attenuation the second beam will propagate further than the first beam. A third beam will propagate even farther, and so on. A sequence of beams rapidly projected along the same path will therefore tunnel into the atmosphere much deeper than otherwise possible with only one beam. Such a rapid sequence of beams can be obtained by a parallel arrangement of Marx generators to be discharged in a programmed sequence by triggered spark gap switches over the same Blumlein line and discharge diode.

I define the ratio $T_{0} / T=\kappa$ where $T_{0}$ is the air temperature under normal conditions and $T$ the temperature in the channel heated by the beam. For $T \sim 10^{4} \mathrm{~K}, \kappa \sim 3 \times 10^{-2}$. With a beam channel volume of $\pi r^{2} l_{0} \sim 10^{3} \mathrm{~cm}^{3}$ a beam energy $\epsilon \sim 10^{4} \mathrm{~J}$ would raise the temperature to $T \sim 10^{4} \mathrm{~K}$. The first beam pulse will penetrate the distance $\delta_{1}=l_{0}$. The second beam will add to this the penetration length $\delta_{2}=$ $(1-\kappa) l_{0}$, the third will add $\delta_{3}=(1-\kappa) \delta_{2}=(1-\kappa)^{2} l_{0}$, and the $n$th beam will add $\delta_{n}=(1-\kappa) \delta_{n-1}=(1-\kappa)^{n-1}$ $l_{0}$. The total penetration length is therefore given by

$$
l_{n}=\sum_{n=1}^{n} \delta_{n}=l_{0} \sum_{n=1}^{n}(1-\kappa)^{n-1}=\left[1-(1-\kappa)^{n}\right] l_{0} / \kappa .
$$

For $n \rightarrow \infty, l_{\infty}=l_{0} / \kappa$. For $l_{0}=2.8 \times 10^{4} \mathrm{~cm}$ and $\kappa=$ $3 \times 10^{-2}, l_{\infty} \sim 10 \mathrm{~km}$. For $n=10$ pulses the length would be $l_{\text {to }}=2.4 \mathrm{~km}$. In this case each beam pulse delivers $\sim$ $10^{4} \mathrm{~J}$ such that the total energy delivered in ten subsequent pulses, with a time lapse of $\sim 10^{-6} \mathrm{~s}$ between the pulses, is $\sim 10^{5} \mathrm{~J}$. The total time to discharge ten subsequent pulses is $\sim 10 \times 10^{-6} \mathrm{~s}=10^{-5} \mathrm{~s}$ and is short enough to prevent the beam channel from being destroyed by either heat conduction or wind motion. A capacitor bank of $10^{5} \mathrm{~J}$ has an approximate weight of several tons. The capacitor bank could be thus mounted on a heavy truck or airplane and charged up by a combustion engine, making the electron beam machine mobile, a desirable requirement if the beam is to be employed for cloud or weather modification.

This method promises the following: (1) It permits the disposal of a large negative electric charge into a chosen volume of the atmosphere or of a cloud. (2) The intense relativistic electrons release within the atmosphere a large $\mathrm{X}$-ray flash producing many pairs of positive and negative ions. (3) The beams create within the atmosphere channels of high electrical conductivity.

The implications of these effects to potential cloud and weather modification are manifold. The most important ones seem to be: (1) The local release of electric charges can result in greatly increased electric fields within a certain cloud region leading to enhanced cloud droplet coalescence resulting in increased precipitation. (2) The release of electric charges in an updraught may accelerate the thundercloud electrification by the Vonnegut-feedback mechanism ${ }^{2}$ and conceivably in this way also increase precipitation. (3) The creation of a large number of ion pairs will increase the electric conductivity of the air which could be used for lightning supression. (4) The creation of long channels of high electrical conductivity could be used to trigger artificially cloud-cloud or cloud-ground discharges.

This work was supported in part by a grant from the US National Science Foundation.

\section{F. WINTERBERG}

Laboratory of Atmospheric Physics,

Desert Research Institute,

University of Nevada System,

Reno, Nevada 89507

Received November 10, 1973.

1 Vonnegut, B., and Moore, C. B., in Recent Advances in Atmospheric Electricity (edit. by Smith, L. G.), 317-331. (Pergamon Press, New York, London, Paris, and Los Angeles, 1958).

${ }^{2}$ Vonnegut, B., Proc. Conf. Atmos. Electricity, 169-181, (Air Force Cambridge Res. Lab., Cambridge, Massachusetts, 1955). 